\title{
Changes in UsnRNA biosynthesis during rat liver regeneration
}

\author{
Rabindranath Ray, Chinmay K. Panda, Bimal K. Chakraborty, \\ Sheela Mukherji, ${ }^{1}$ Kanakendu Chaudhury ${ }^{2}$ and Jayashree \\ Roychoudhury
}

Department of Biochernistry, ${ }^{\prime}$ Department of Biophysics, Chittaranjan National Cancer Institute, 37, S.P. Mukherjee Road, Calcutta, India; ${ }^{2}$ Molecular Immunology laboratory, Division of Gastroentrology and Hepatology, University of Medicine and Dentistry of New Jersey, NJ, USA

Received 17 June 1993; Accepted 15 November 1993

\begin{abstract}
Partial hepatectomy (P.H.) induces a partially synchronized growth response of liver under normal regulation of growth. In this phase changes in cellular morphology, radial distribution pattern of cells and other biological as well as major biochemical changes are well documented [24]. Here, we have shown that the cellular content of UsnRNAs altered during this proliferative phase as well. The level of spliceosomal UsnRNAs (U1, U2, U4-U6) gradually decreased by $30-50 \%$ upto $48 \mathrm{hrs}$ of P.H. followed by gradual increase to reach the normal level within one month of P.H. The U3 snRNA level on the other hand, was nearly equal to that in normal liver at 48 hrs of P.H. but in 24 and 72 hrs of P.H. its level was high ( 4 fold) in contrast to that in other UsnRNAs. Thus, it is clear from our data that the level of all the six UsnRNAs decreased during $48 \mathrm{hrs}$ of P.H. compared to that after first $24 \mathrm{hrs}$. This has been correlated in the kinetics of UsnRNAs' synthesis (in terms of labelling) in isolated hepatocytes, where the rate of labelling of all the six UsnRNAs increased $20-30 \%$ in 24 hrs regenerating hepatocytes (R.H.) followed by sharp decrease by $30-50 \%$ within next $24 \mathrm{hrs}$, compared to that in the normal hepatocytes. But from 72 hrs onwards in R.H. the rate of labelling of all the six UsnRNAs again increased by 30-50\% (compared to that in normal hepatocytes) followed by decrease of their labelling-rate to reach the normal level in R.H. within one month of P.H. Thus, it may be concluded that the changes in UsnRNAs' level during the proliferative phase of liver regeneration may be either due to the alteration in the rate of synthesis (in terms of labelling) or along with it differential turn over rate; this phenomenon may have some consequences with the regenerative process of liver. (Mol Cell Biochem 141: 71-77, 1994).
\end{abstract}

Key words: regenerating rat liver, UsnRNAs, UsnRNA profiles, kinetics of UsnRNAs' labelling

\section{Introduction}

Small nuclear RNAs (SnRNAs) of the U family (U1 to U10) are evolutionarily conserved, metabolically stable, nonpolyadenylated RNA molecules present in the nu- clei of eukaryotic cells [1,2]. The most abundant group include U1 through U6 snRNAs of which the first five contain hypermethylated guanosine cap at the $5^{\prime}$ end 
and is transcribed by RNA polymerase II (pol II) $[3,4]$. The U6, on the other hand, has $\gamma$ monomethyl phosphate at the 5 ' end [5] and is transcribed by RNA polymerase III (pol III) [4]. Except U3, the five nucleoplasmic UsnRNAs are involved in splicing of pre-mRNA [6]. But nucleolar UsnRNA i.e. U3, is involved in pre-rRNA processing [7-9]. So, the major biochemical role of these UsnRNAs are to process pre-mRNA and pre-rRNA. Moreover, studies on the metabolism of UsnRNAs in proliferating/differentiating cells indicated major quantitative alteration in UsnRNA level with no new appearence or disappearence of any individual snRNA species [10-14]. Preferential expression of certain forms of U1 snRNA during mouse embryo development $[15,16]$, differential accumulation of U1 and U4 snRNA through embryogenesis in xenopus [17], over expression of U6 snRNA during xenopus development [18] and tissue specific expression of U4 snRNA in Chicken [19] have also been reported. The $3^{\prime}$ end formation of spliceosomal U6 snRNA depends on specificity of the organism and also on the stage of development [20]. It is also reported that U5 snRNA after transfection, can transform primary cells in vitro [21]. Thus, quantitative alteration in UsnRNA biosynthesis during cellular proliferation/ differentiation, embryonic development, or transforming activity of some particular UsnRNA indicate that any control of cell growth may have some regulation through altered UsnRNA biogenesis.

To study the UsnRNA metabolism during rapid cellular proliferation under normal regulation of growth, we have taken regenerating rat liver as a tool. Ninety per cent by volume ( $50 \%$ by number) of the adult liver is composed of parenchymal cells (hepatocytes) and 5\% by volume ( $40 \%$ by number) is composed of nonparenchymal cells (ductal cells, endothelial cells and Kuppfer cells) [22]. The removal of $70 \%$ of the liver by partial hepatectomy (P.H.) induces a partially synchronized growth response which involves a hypertrophy phase (lasting for 12 to $16 \mathrm{hr}$ ), a replicative phase in which $\sim 84 \%$ of the hepatocytes undergo DNA synthesis (peak at $24 \mathrm{hrs}$ ) and a rapid-cell-division phase, when nearly $70 \%$ of the hepatocytes undergo their first set of cell division within $30 \mathrm{hr}$ of P.H.; by $72 \mathrm{hrs}$ of P.H. about $90 \%$ of the hepatocytes divide at least once [23,24]. On the contrary, the nonparenchymal cells undergo DNA synthesis around $42 \mathrm{hr}$ of P.H. [22]. Thus, in the present work, we want to study the UsnRNA metabolism in the proliferative phase ( 24 to $72 \mathrm{hrs}$ ) of liver regeneration when most of the biochemical changes take place.

\section{Materials and methods}

Bovine Serum Albumin (BSA) fraction V and collagenase type IV, were from Sigma Chemical Company, USA. HAM's F-12 medium was from GIBCO-BRL, USA. ${ }^{3} \mathrm{H}$-Orotic acid $(10000 \mathrm{mCi} / \mathrm{mmole})$ was from BARC, Trombay, India. Other chemicals were of analytical grade.

Animals, partial hepatectomy and perfusion of liver

Male albino rat weighing 180-200 gms were routinely used for partial hepatectomy. Seventy percent hepatectomy was achieved by removing the left lobe and the median lobe of liver according to the method of Higginis and Anderson [25]. Restorative growth of liver took place by a rapid-cell-division phase which continued from 24 to $72 \mathrm{hrs}$ of partial hepatectomy [24]. Rats were sacrificed at $24 \mathrm{hrs}$ interval upto $96 \mathrm{hr}$ and also after one month of P.H. Sham-operated normal rat livers were used as control. Livers were perfused through the portal vein by perfusion buffer $\left(0.08 \mathrm{M} \mathrm{NaH} \mathrm{HO}_{4} / \mathrm{Na}_{2} \mathrm{HPO}_{4}\right.$, $\mathrm{pH} 7.4)$ containing heparin (10 units/ml) to get rid of blood contamination.

\section{Subcellular fractionation, RNA extraction and UsnRNA} fractionation

Nuclei and cytoplasmic supernatant were prepared from perfused rat liver as described in [26] with slight modification. Perfused liver was passed through the metal net, single cells were washed with PBS (Phosphate Buffered Saline) at $500 \mathrm{rpm}$ for $5 \mathrm{~min}$ at room temperature. The cells were suspended in 10 vols. (10 volumes/ $\mathrm{gm})$ of homogenisation buffer $(2.5 \%$ Citric acid, $0.2 \%$ Triton-X-100), kept in ice for $10 \mathrm{~min}$ and homogenised with 10-15 strokes in Dounce homogeniser fitted with B pestle. The suspension was then centrifuged at $1,500 \mathrm{~g}$ for $10 \mathrm{~min}$ at $4^{\circ} \mathrm{C}$. The supernatant fraction (SF) was stored in ice. The pellet containing the crude nuclei was washed once with the homogenisation buffer. The pellet was suspended in suspension buffer ( $2.5 \%$ Citric acid) and passed through a cushion containing $2.5 \%$ Citric acid, $15 \%$ glycerol at $1,500 \mathrm{~g}$ for $15 \mathrm{~min}$ at $4^{\circ} \mathrm{C}$. Pellet containing the purified nuclei was washed once with the suspension buffer and kept in ice. The 'SF' was centrifuged at $15,000 \mathrm{~g}$ for $15 \mathrm{~min}$ at $4^{\circ} \mathrm{C}$. The pellet (SF-P) 
and the supernatant fraction (SF-1) were saved and kept in ice. RNAs were extracted from the purified nuclei, whole cells and also from 'SF-P' by using guanidine isothiocynate, according to the method of Chomczynski and Sacchi [27] and from 'SF-1' by Phenol: Chloroform extraction.

For electrophoretic analysis, RNAs were dissolved in $80 \%$ deionised formamide, heated to $85^{\circ} \mathrm{C}$ for $3 \mathrm{~min}$, chilled immediately in ice and electrophoresed on $12 \%$ polyacrylamide - $7 \mathrm{M}$ Urea gel as described in [28]. The gel was ethidium bromide stained and photographed. Then the gel was silver stained [29], dried and scanned densitometrically at $410 \mathrm{~nm}$ using a densitometric scanner (SHIMADZU MODEL-CS-9000). We have observed that most of the cytoplasmic RNAs were present in the SF-P fraction compared to the SF-1 (data not shown).

Identification of UsnRNA bands on the scanning profile were accomplished by comparing their mobilites with that of known cytoplasmic marker RNAs namely $4 \mathrm{~S}, 4.5 \mathrm{~S}, 5 \mathrm{~S}$ and $5.8 \mathrm{~S}$, as well as by direct comparison to snRNAs isolated from whole nuclei [30, 31]. Quantitation by densitometry after silver staining was done under the condition that has given a linear response with the RNA concentration [29]. Relative abundance of each UsnRNA was calculated by normalizing the areas of the peaks with respect to that of corresponding $5 \mathrm{~S}$ rRNA [28, 32-34].

\section{In vitro labelling of UsnRNAs in isolated hepatocytes}

Viable hepatocytes were isolated from sham-operated normal liver and partially hepatectomized rat liver (as described earlier) according to the method of Öbrink [35]. The abdomen of the rat under light ether-anaesthesia was opened and $0.25 \mathrm{ml}$ of heparin $(5000 \mathrm{IU} / \mathrm{ml})$ was injected through the iliolumber vein. The $\mathrm{Ca}^{2+}$ free perfusion buffer $(0.14 \mathrm{M} \mathrm{NaCl}, 0.0067 \mathrm{M} \mathrm{KCl}, 0.01 \mathrm{M}$ HEPES, $\mathrm{pH}$ 7.4) was passed through the portal vein at a flow rate of $20 \mathrm{ml} / \mathrm{min}$ for a period of $20 \mathrm{~min}$. The collagenase buffer $(0.0066 \mathrm{M} \mathrm{NaCl}, 0.0067 \mathrm{M} \mathrm{KCl}, 0.0047 \mathrm{M}$ $\mathrm{CaCl}_{2}$. 0.1 M HEPES pH 7.6 containing $15 \mathrm{mg} / \mathrm{ml}$ of BSA and $0.5 \mathrm{mg} / \mathrm{ml}$ collagenase) was then passed through the portal vein as before with recirculation for $10 \mathrm{~min}$. Cells were liberated by gently shaking the perfused liver in cell suspension buffer $(0.14 \mathrm{M} \mathrm{NaCl}$, $0.0067 \mathrm{M} \mathrm{KCl}, 0.01 \mathrm{M} \mathrm{HEPES}$, pH 7.4 containing $7.5 \mathrm{mg} / \mathrm{ml}$ of BSA). The cell suspension was centrifuged at $500 \mathrm{rpm}$ for $2 \mathrm{~min}$ at room temperature, followed by washing the cell pellet twice with the same buffer. Viable cells, in suspension, was found to be 75 to $85 \%$ as determined by Trypan blue exclusion method.

Isolated hepatocytes, suspended at a concentration of $10^{6} \mathrm{cells} / \mathrm{ml}$ in HAM's F-12 medium buffered with $25 \mathrm{mM}$ HEPES-0.014 $\mathrm{M} \mathrm{NaHCO}_{3}$, pH 7.4 containing $15 \%$ (V/V) FCS and ${ }^{3} \mathrm{H}$-Orotic acid $\left(10 \mathrm{Ci} / 10^{6} \mathrm{cells} / \mathrm{ml}\right)$, were incubated at $37^{\circ} \mathrm{C}$ with constant shaking over a period of $3.5 \mathrm{hr}$. The cell suspension $(5 \mathrm{ml}$ at each time point) was collected at $1 \mathrm{~h}$ interval upto $3 \mathrm{hr}$ and also after $3.5 \mathrm{hr}$ of incubation. Cells were centrifuged at 5000 rpm for $2 \mathrm{~min}$ at room temperature and washed twice with PBS. Whole cellular RNAs were extracted from these cell pellets and fractionated on polyacrylamide gel by electrophoresis as described before. The gel was ethidium bromide stained and photographed. Fluorography of the gels were done on KODAK X-OMAT AR-5 film through $1 \mathrm{M}$ Sodium Salicylate impregnation according to the method of Chamberlin [36]. Identification of UsnRNAs' bands on the fluorogram was done as described in silver stained gel. Bands in the X-ray film and in the negative of ethidium bromide stained gel were densitometrically scanned as before. Quantitation by densitometry was done following the procedure of Choudhury et al. [28] to give a linear response both with $\mathrm{X}$-ray film and the negative of ethidium bromide stained gel. To study the kinetics of labelling of each of the six UsnRNAs during liver regeneration, area of each labelled UsnRNA band was normalised to that of the total unlabelled area of the corresponding 5 S rRNA [28, $32-$ $34]$ and plotted against different period of incubation.

\section{Results}

\section{Alteration in the profile of UsnRNA during liver regeneration}

We have studied alterations of UsnRNA profiles during the proliferative phase of liver regeneration i.e. from 24 to $96 \mathrm{hrs}$ of P.H. It was apparent from ethidium bromide stained gel (gel not shown) that there was some alteration in UsnRNA content at least at 48 hrs of P.H. The gel was then silver stained for densitometric scanning. It was revealed from Fig. 1 that there was gradual decrease in abundance of the five spliceosomal UsnRNAs (U1, U2, U4-U6) upto 48 hrs of P.H. At this phase (i.e. after 48 hrs of P.H.) the abundance of U1 and U2 snRNAs decreased nearly $30 \%$, while in case of U4-U6 snRNAs the decrease in abundance was about $50 \%$. Then their abun- 

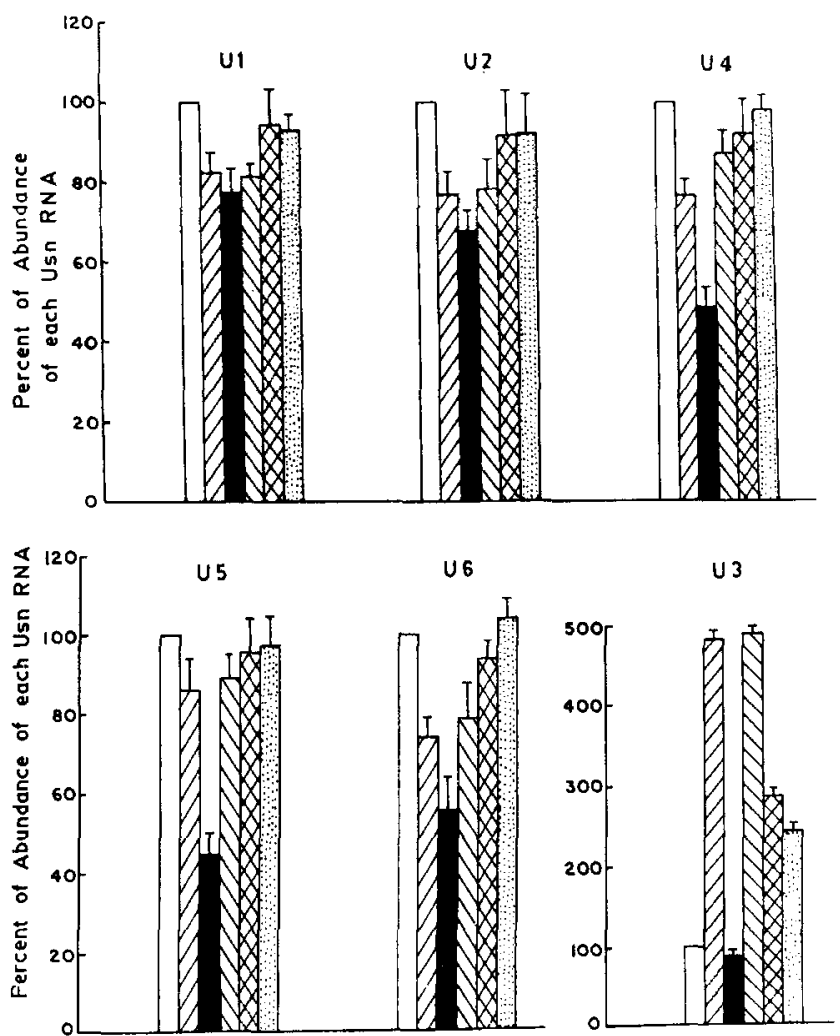

Fig. 1. Profile of each UsnRNAs in sham-operated normal liver and livers of $24 \mathrm{hr}, 48 \mathrm{hr}, 72 \mathrm{hr}, 96 \mathrm{hr}$ and one month of P.H. Silver stained gel was scanned densitometrically. Relative abundance of each UsnRNAs was obtained by normalizing the area of each UsnRNA peak in the silver stained gel with that of corresponding $5 \mathrm{~S}$ rRNA. Percent of abundance was calculated by taking the relative abundance of each UsnRNA in Sham-operated normal as control i.e. percent of abundance of each UsnRNA is the relative abundance of each UsnRNA in P.H. liver sample, divided by the relative abundance of each UsnRNA in normal liver sample, multiplied by $100 . \square$ normal; 24 hrs of P.H.; 48 hrs. of P.H.; 72 hrs of P.H.; 96 hrs. of P.H.; 图 one month of P.H.;

dance gradually increased and reached normal level within one month of P.H. On the contrary, the abundance of U3 snRNA was nearly same as in normal after $48 \mathrm{hrs}$ of P.H. Its abundance sharply increased about 4 fold compared to that in normal after $24 \mathrm{hrs}$ and $72 \mathrm{hrs}$ of P.H. Thereafter, the level of U3 gradually decreased until one month of P.H. Thus, during proliferative phase of liver regeneration, the abundance of UsnRNAs altered considerably.

\section{Kinetics of UsnRNAs' labelling during liver regeneration}

Rate of UsnRNAs' labelling during proliferative phase of liver regeneration i.e 24 to $96 \mathrm{hrs}$ of P.H. was studied in vitro in isolated hepatocytes, as described in materials and methods. During in vitro culture of isolated hepato- cytes (from regenerating rat liver), it has been observed that UsnRNAs could be significantly labelled with radioactive orotic acid only after $1 \mathrm{hr}$ of incubation at $37^{\circ} \mathrm{C}$ (data not shown). The rate of labelling of all the six UsnRNAs reached plateau after 3 hrs of incubation at $37^{\circ} \mathrm{C}$ (Fig. 2). At the first replicative phase of liver regeneration i.e after $24 \mathrm{hrs}$ of P.H., the rate of labelling of all the six UsnRNAs increased $20-30 \%$ compared to that in normal hepatocytes. But after 48 hrs of P.H. the levels of labelled UsnRNAs sharply decreased to approximately 30 to $50 \%$ with respect to that in normal hepatocytes (Fig. 2). However, during last phase of proliferation of liver regeneration, i.e 72 hrs after P.H. the levels of all the six labelled UsnRNAs considerably increased (nearly $30-50 \%$ ), followed by gradual decrease in their rates of labelling to restore normal rate within one month of P.H. Therefore, in the proliferative phase of liver regeneration the biosynthetic rate (in terms of labelling) of all the six UsnRNAs are not same. The upregulation of their rate of synthesis (in terms of labelling) after 24 and $72 \mathrm{hrs}$ of P.H. along with downregulation after $48 \mathrm{hrs}$ of P.H. might have some consequences with the regenerative process of liver growth.

\section{Discussion}

The major metabolic alterations during liver regeneration after P.H. have been reviewed in great details [23, 24]. The total RNA concentrations both in the nucleus and in the cytoplasm (including cytoplasmic poly $\mathrm{A}^{+}$ mRNA) reached to a maximum level within $24 \mathrm{hrs}$ of P.H., but their level decreased sharply within $48 \mathrm{hrs}$ of P.H. Whereas the nuclear poly $\mathrm{A}^{+}$mRNA level remained unaltered during this period $[37,38]$. Though, there was no remarkable change in liver specific mRNA transcription rate or its concentration within first $24 \mathrm{hrs}$ of P.H. a definite increase in concentration of actin mRNA level and higher transcription rate for tubulin mRNA within 24 hrs of P.H. have been reported [39]. That, the rate of pre-rRNA synthesis and ribosomal protein synthesis did not alter during the proliferative phase of liver regeneration after P.H. have been well documented; on the contrary, the ribosome biosynthesis increased considerably during this phase of liver regeneration [39-41]. However, the activation of pre-rRNA synthesis in regenerating rat liver due to increased RNA Polymerase I activity has also been reported [42]. But it has been observed from Fig. 1 that the profile of UsnRNAs changed considerably during the proliferative phase of 

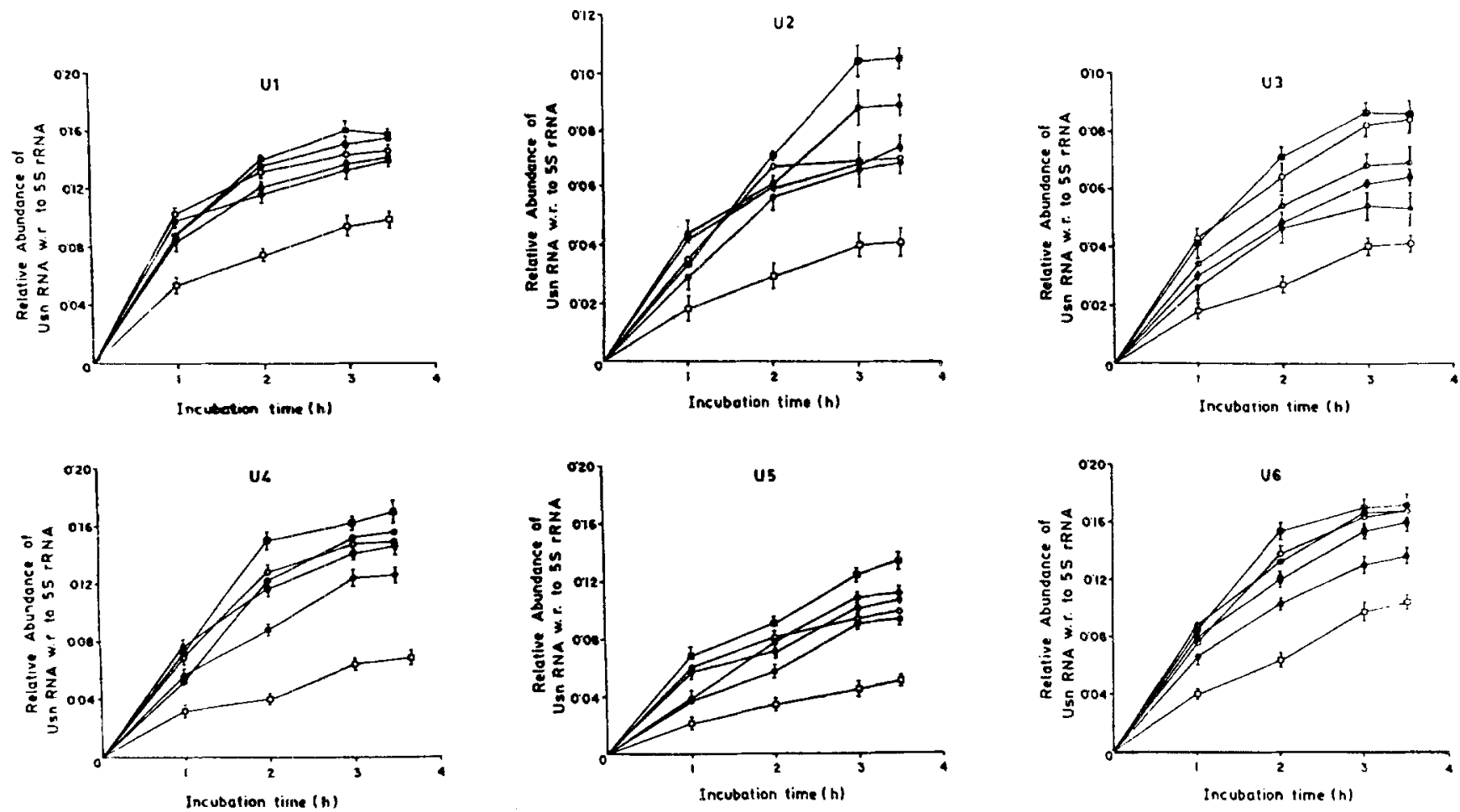

Fig. 2. Kinetics of each UsnRNA's labelling in isolated hepatocytes from sham-operated normal liver and livers of $24 \mathrm{hr}, 48 \mathrm{hr}, 72 \mathrm{hr}, 96 \mathrm{hr}$ and one month of P.H. Relative abundance of each labelled UsnRNA at each time point was obtained by normalizing the area of each labelled UsnRNA band with that of total 5S rRNA in the ethidium bromide stained gel. (Area of each RNA band was calculated by densitometric scanning of either fluorogram or the ethidium bromide stained gel.) -0 normal; $\bigcirc-\bigcirc 24$ hrs of P.H.; $\square-\square 48$ hrs of P.H.; $\square-\square 2$ hrs of P.H.; D-D 96 hrs of P.H.; - one month of P.H.

liver regeneration. The levels of five UsnRNAs (except U3) which constitute the active spliceosomal complex gradually decreased ( 30 to $50 \%$ ) upto 48 hrs of P.H. followed by sharp increase to reach the normal level within one month of P.H. In contrast, U3 snRNA increased 4 fold around 24 hrs of P.H. followed by a sharp decrease within next 24 hrs (Fig. 1). But after 72 hrs of P.H., the U3 snRNA's level again increased sharply (4 fold) compared to other UsnRNA's level. Thus, it is evident from Fig. 1, that the level of all the six UsnRNAs decreased sharply during $48 \mathrm{hrs}$ of P.H. compared to the first $24 \mathrm{hrs}$. So the changes in the total UsnRNAs' pool, during the proliferative phase of liver regeneration might be either due to alteration in the rate of synthesis or in the turn over rate.

It is evident from Fig. 2 that the kinetics of spliceosomal UsnRNAs' synthesis (in terms of labelling) is highly correlated with that of the cytoplasmic poly $\mathrm{A}^{+}$mRNA synthesis during liver regeneration [38]. However, the rate of labelling of the five UsnRNAs in first $24 \mathrm{hrs}$ regenerating hepatocytes (R.H.) increased nearly 20 $30 \%$, yet the total cellular contents of these UsnRNAs was low $(\sim 20 \%)$ (Fig. 1) as compared to that in normal liver. This might be due to the faster turnover rate of these UsnRNAs during this phase of liver regeneration. Whereas, during the next 24 hrs of liver regeneration i.e $48 \mathrm{hrs}$ of P.H. the rate of labelling in R.H. decreased approximately $30-50 \%$ along with the decrease $(\sim 30-$ $50 \%$ ) in total cellular content of these UsnRNAs. Thus, in this phase of liver regeneration, the turnover rate of these UsnRNAs was not exactly same as that in first $24 \mathrm{hrs}$ of liver regeneration. Because, if the turnover rate of these UsnRNAs in 48 hrs regenerating liver was same as that in $24 \mathrm{hrs}$, then the total cellular contents of these UsnRNAs in 48 hrs regenerating liver would be much lower than the level we have observed. But during regeneration after $72 \mathrm{hrs}$ of P.H. both the rate of labelling and total cellular content of these UsnRNAs were nearly comparable as that in first $24 \mathrm{hrs}$ sample. This might be due to the fact that during these phases of liver regeneration, major portion of the hepatocytes might be in the same stage of cell division $[23,24]$. Higher abundance of U3 snRNA, which is involved in pre-rRNA processing [7-9] has been well documented in two developmental phases of xenopus: a) during Oocyte maturation and b) late embryogenesis [43]. We have shown that total pool 
of U3 snRNA at 24 and 72 hrs of P.H. was different from that of all the five spliceosomal UsnRNAs though their rates of labelling were similar (Fig. 1 and Fig. 2). It seems likely, that during these phases of liver regeneration the rate of turn over of U3 snRNA may be low as compared to that of other nucleoplasmic UsnRNA. However, after $48 \mathrm{hrs}$ of P.H. the total cellular pool of U3 snRNA decreased to the normal level in comparison to that in first $24 \mathrm{hrs}$ regenerating liver. This might be either due to lowering (30-50\%) in rate of labelling in $48 \mathrm{hrs} \mathrm{R.H.} \mathrm{or}$ along with it a higher turn over rate in this phase of liver regeneration.

It is evident from our data that the UsnRNAs were accumulated in different ways in different phases of liver regeneration, suggesting that the UsnRNAs may be differentially regulated according to specific cellular requirements or function. It has also been reported that some of the UsnRNAs' proportions are altered differentially in both stationary phase and terminally differentiated cells [14]; certain UsnRNAs may be involved in the production of mRNA and are coordinately regulated with respect to the alterations in resting/differentiating cellular-gene activity besides other factors involved [44]. The UsnRNAs are transcribed by two different RNA polymerases: U1 to U5 snRNAs by pol II and U6 by pol III [4], although their mode of synthesis appears to be similar. The promoter region of these UsnRNAs are different from other pol II and pol III transcribed RNA promoters [45]. Several cis acting elements (e.g Sph motiff, Octamer motiff, GC box, LINES sequence etc.) similar to that of mRNA genes have been characterised in different UsnRNAs along with some transacting factors [46]. It is quite evident that these cis-acting elements have some role in cellular proliferation, differentiation as well as tissue specific gene expression [47]. Besides, it has been shown that these UsnRNA enhancer elements can act in a variety of cis-linked configurations to activate gene transcription synergistically [46]. Thus, the differential regulation of these UsnRNAs' transcription during the proliferative phase of liver regeneration might be controlled by all these elements.

\section{Acknowledgements}

We are grateful to Drs. A. Dasgupta, S. Saha, R.K. Chatterjee, A. Chatterjee, and Mr. K. Ray, for their help and valuable suggestions during the work. This investigation was supported by Junior Research fellowship from ICMR, India, awarded to R. Ray.

\section{References}

1. Reddy R, Busch H: Small nuclear RNAs: RNA sequences, structure, and modifications. In: Birnstiel ML (ed.) Structure and function of major and minor small nuclear ribonucleoprotein particles. Springer-Verlag, Heidelberg, 1988, pp 1-37

2. Fillipowicx W, Kiss T: UsnRNA genes, UsnRNAs, and UsnRNPs of higher plant. Molec Biol Rep 14: 125-130, 1990

3. Mattaj IW: Cap trimethylation of UsnRNA is cytoplasmic and is dependent on UsnRNP protein binding. Cell 46: 905-911, 1986

4. Dahlberg JE, Lund E: The genes and transcription of the major small. nuclear RNAs. In: Birnstiel ML (ed.) Structure and function of major and minor small nuclear ribonucleoprotein particles. Springer-Verlag. Heidelberg, 1988, pp 38-70

5. Singh R, Reddy R: $\gamma$ Monomethyl phosphate: A cap structure in spliceosomal U6 small nuclear RNA. Proc Natl Acad Sci USA 86: 8280-8283, 1989

6. Guthrie C: Messenger RNA splicing in yeast: clues to why the spliceosome is a ribonucleoprotein. Science 253:157-163, 1991

7. Savino R, Gerbi SA: In vivo disruption of Xenopus U3 snRNA affects ribosomal RNA processing. EMBO J 9(7): 2299-2308. 1990

8. Kass S, Tyc K, Steitz JA, Sollner-Webb B: The U3 small nucleolar ribonucleoprotein functions in the first step of preribosomal RNA processing. Cell 60: 897-908, 1990

9. Hughes JMX, Ares Jr M: Depletion of U3 small nucleolar RNA inhibits cleavage in the 5 ' external transcribed spacer of yeast preribosomal RNA and impairs formation of $18 \mathrm{~S}$ ribosomal RNA. EMBO J 10(13): 4231-4239, 1991

10. Howard EF, Stubblefied E: Low molecular weight nuclear RNA in phytohaemagglutinin - treated and untreated lymphocytes. Exp Cell Res 70: 460-462, 1972

11. Hellung-Larsen P, Tyrsted G, Fredriksen S: Low-molecular weight nuclear RNA components in human lymphocytes cultured without or with phytohaemagglulinin. Exp Cell Res 80: 393-404, 1973

12. Howard EF: Lowmolecular weight RNA in human lymphocyte. A comparison of PHA-treated and control cells. Exp Cell Res 82: 280-286, 1973

13. Rein A, Penman S: Synthesis of the small molecular weight monodisperses nuclear RNAs in contact-inhibited cell cutters. J Cell Physiol 79: 453-456, 1973

14. Kuncio G, Goldstein L: Small nuclear RNAs in cellular growth and differentiation I: Metabolic alterations seen in friend erythroleukemic cells. J Cell Physiol 109: 235-241, 1981

15. Bach M, Krol A, Lührman R: Structure-probing of U1 snRNPs gradually depleted of the U1 specific proteins A, C and 70K. Evidence that $A$ interacts with developmentally regulated mouse $U 1$ snRNA variants. Nucl Acids Res 18: 449-457, 1990

16. Lund E, Kahan B, Dahlberg JE: Differential control of U1 smal] nuclear RNA expression during mouse development. Science 229: 1271-1274, 1985

17. Lund E, Dahlberg JE: Differential accumulation of U1 and U4 small nuclear RNAs during Xenopus development. Genes and Dev 1: $39-46,1987$

18. Hamm J, Mattaj IW: An abundant U6 snRNP found in germ cell and embryos in Xenopus levis. EMBO J 8: 4179-4187, 1989

19. Korf GM, Botros IW, Stumph WE: Development and tissue spe- 
cific expression of U4 small nuclear RNA genes. Mol Cell Biol 8: 5566-5569, 1988

20. Lund E, Dahlberg JE: Cyclic 2', 3'-phosphate and non-templated nucleotides at $3^{\prime}$ end of spliceosomal U6 small nuclear RNA's. Science 255: 327-330, 1992

21. Hamada K, Kumazaki T, Mizuno K, Yokoro K: A small nuclear RNA, U5, can transform cells in vitro. Mol Cell Biol 9: 4345-4356, 1989

22. Grisham JW: A morphologic study of deoxyribonucleic acid synthesis and cell proliferation in regenerating rat livers; autoradiography with Thymedin $-{ }^{3} \mathrm{H}$. Cancer Res 22: 842-849, 1962

23. Bucher NI, Malt RA: Regeneration of liver and kidney. Little Brown and Co. Biston, 1971, p 79

24. Fausto N: Messenger RNA in regenerating liver: implication for the understanding of regulated growth. Mol Cell Biochem 59:131147,1984

25. Higginis GM, Anderson RM: Experimental pathology of the liver. Restoration of the liver of the white rat following partial surgical removal. A M A Arch Pathol 12: 186-202, 1931

26. Reinner JJ, Busch H: Transcriptional and post-transcriptional modulation of cytoplasmic ribonuclic acids in regenerating liver and Novikoff hepatoma. Biochemistry 19: 833-841, 1980

27. Chomczynski P, Sacchi N: Single step method of RNA isolation by acid guanidium thiocyanate - phenol - chloroform extraction. Anal Biochem 162: 156-159, 1987

28. Choudhury K, Choudhury I, Jones RW, Thirunavukkarasu C, Eliceiri GL: Metabolism of U6 RNA species in nonirradiated and UV-irradiated mammalian cells. J Cell Physiol 137: 529-536, 1988

29. Blum H, Beier H, Gross HJ: Improved silver staining of plant proteins, RNA and DNA in polyacrylamide gel. Electrophoresis 8 : 93-99, 1987

30. Guialis A, Dangli A, Sckeris CE: Distribution of snRNP complexes in rat liver nuclear extracts: Biochemical and immunochemical analysis. Mol Cell Biochem 76: 147-162, 1987

31. Reddy R, Gupta S: Compilation of small RNA sequences. Nucl Acids Res 18 suppl: 2231-2235, 1990

32. Thirunavukkarasu C. Choudhury K, Ninichuck AJ, Choudhury I, Eliceiri G: Effect of ultraviolet light on the expression of genes for human U1 RNA. J Cell Physiol 137: 55-64, 1988
33. Choudhury K, Choudhury I, Eliceiri G: Metabolism of small RNAs in cultured human cells. J Cell Physiol 138: 433-438, 1989

34. Eliceiri BP, Choudhury K, Scott QO, Eliceiri G: Ultraviolet lightinduced inhibition of small nuclear RNA synthesis. J Cell Physiol 138: 586-592, 1989

35. Öbrink B: Hepatocytes - collagen adhesion, Methods in Enzymology 77: 513-529, 1982

36. Chamberlin JP: Fluorographic detection of radioactivity in polyacrylamide gels with the water soluble fluor, sodium salicylate. Anal Biochem 98: 132-135, 1979

37. Walker PR, Whitfield JF: Regulation of the pre-replicative changes in the synthesis and transport of messenger and ribosomal RNA in regenerating livers of normal and hypocalcemic rats. $J$ Cell Physiol 108: 427-437, 1981

38. Atryzek N, Fausto N: Accumulation of polyadenylated mRNA during liver regeneration. Biochemistry 18: 1281-1287, 1979

39. Friedman JM, Chung EY, Darnel Jr JE: Gene expression during liver regeneration. J Mol Biol 179: 37--53, 1984

40. Chaudhuri S, Luberman I: Control of ribosome synthesis in normal and regenerating liver. J Biol Chem 243: 29-33, 1968

41. Warner JR: The assembly of ribosomes in eukaryotes. In: Nomura M, Tissieres A, Lengyel P (eds) Ribosomes. Cold Spring Harbour Laboratory, Cold Spring Harbour, New York, 1974, pp 417-488

42. Nokolov EH, Nankova BB, Dabeva MD: Activated ribosomal RNA synthesis in regenerated rat liver upon inhibition of protein synthesis. Molec Biol Rep 15: 45-52, 1991

43. Caizergues-Ferrer M, Mathieu C, MarioHini P, Amalric F, Amaldi F: Fibrillarin and U3 RNA expression during Xenopus Oogenesis and embryo development. Molec Biol Rep 14: 107-108, 1990

44. Marks PA, Rifkind RA: Erythroleukemic differentiation. Ann Rev Biochem 47: 419-448, 1978

45. Das G, Henning D, Wright D, Reddy R: Upstream regulatory elements are necessary and sufficient for transcription of a U6 RNA gene by RNA polymerase III. EMBO J 7: 503-512, 1988

46. Roebuck KA, Szeto DP, Green KP, Fan QN, Stumph WE: Octamer and SPH motifs in the U1 enhancer cooperate to activate U1 RNA gene expression. Mol Cell Biol 10: 341-351, 1990

47. Blau HM: Differentiation requirs continuous active control. Ann Rev Biochem 61: 1213-1230, 1992 